\title{
The concept and realization of high-power laser diodes with multi- stripe-gain distribution
}

\author{
Grzegorz Sobczak, ${ }^{* 1,2}$ Elżbieta Dąbrowska, ${ }^{1}$ Marian Teodorczyk, ${ }^{1}$ Konrad Krzyżak, ${ }^{1}$ Joanna Kalbarczyk, ${ }^{1}$ \\ and Andrzej Maląg ${ }^{1}$ \\ ${ }^{1}$ Institute of Electronic Materials Technology, Wólczyńska 133, 01-919 Warszawa, \\ ${ }^{2}$ Faculty of Physics, Warsaw University of Technology, Koszykowa 75, 00-662 Warszawa
}

Received July 31, 2013; accepted August 08, 2013; published September 30, 2013

\begin{abstract}
Slow axis far-field widening (far-field blooming) is one of the factors which decrease the quality of an emitted beam of high-power laser diodes. This effect is connected with a rising number of maintained lateral optical modes in the laser waveguide. In this paper the design of the gain distribution by the steering of a current flow is presented as a way to restrict the far-field blooming. Numerical and experimental results are presented. An almost constant slow-axis width of optical field distribution in the far-field is obtained up to four times above the threshold current.
\end{abstract}

The implementation of high-power laser diodes (LDs) in many applications requires high stability of an emitted beam. In standard construction of these devices a broad active (BA) stripe is used to obtain high power. Unfortunately, in this solution the quality of the emitted beam in a lateral direction (slow axis) is poor. It is due to the multimode character of the beam and its low stability. One of the symptoms of low stability is the far-field widening (also called: far-field blooming). It is connected with self-heating which leads to variations of the refractive index. The rise of the refractive index with temperature in an active region enhances the waveguide effect. Consequently, higher order lateral modes can appear. It is well known that higher order modes have wider distribution in the far-field [1-3]. This effect is restricted in the Phase Locked Arrays (PLA) of semiconductor lasers [4-6]. In these devices, the BA stripe is replaced by an array of narrow waveguides and the optical fields from the neighboring active stripes are coupled to each other. As a result of the coupling, the optical field forms a set of supermodes propagating over the whole structure. The PLA radiation usually forms two beams located symmetrically with respect to the device optical axis. These beams are highly-stable and nearly diffraction limited. However, the PLA structures prove to be technologically difficult.

In this paper the concept of the BA laser with a limited effect of slow-axis far-field blooming is presented. The numerical results are obtained using the FIMMWAVE program (by Photon Design), which had been successfully

*E-mail: grzegorz.sobczak@itme.edu.pl used in earlier investigations [7-8] to predict the distributions in the near- and far-field (NF, FF respectively). Confirming experimental results are presented as well.

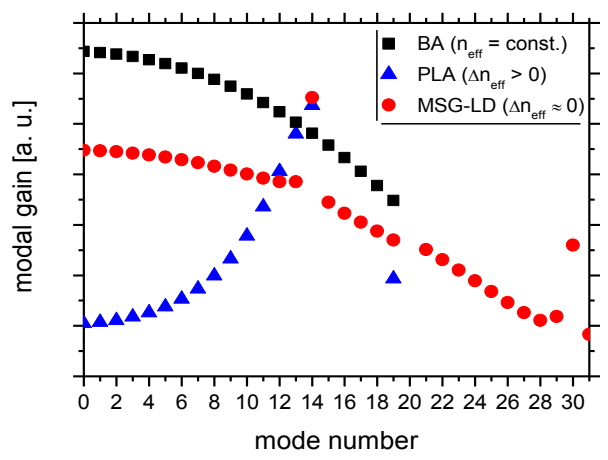

Fig. 1. Simulated modal gain of modes in various LD constructions: (square) standard BA laser diode, (triangle) PLA containing 15 active stripes and (circle) MSG-LD with the gain distribution similar to that of PLA. The total widths of the active regions in PLA and in MSG-LD are similar to the BA width. As it can be seen, the MSG-LD construction enhances the gain of the $14^{\text {th }}$ order lateral mode (the fundamental mode in this notation is the $0^{\text {th }}$-order one due to zero phase-sign changes in optical field distribution)

The idea is to form by ion implantation a laterallyperiodic gain distribution in the active region of LD (here such a construction will be called a multi-stripe-gain laser diode: MSG-LD). The goal is that the inducted variations in the gain distribution should provide a low threshold current $\left(\mathrm{I}_{\mathrm{th}}\right)$ of the higher order lateral mode but should not be able to form supermodes as in the PLA structures. To fulfill these assumptions, the ion implantation should cause only a small difference between the effective indices $\left(\Delta \mathrm{n}_{\text {eff }}\right)$ in implanted $\left(\mathrm{n}_{\text {eff } 2}\right)$ and unimplanted $\left(\mathrm{n}_{\text {eff1 }}\right)$ regions $\left(\Delta \mathrm{n}_{\mathrm{eff}}=\mathrm{n}_{\text {eff } 1}-\mathrm{n}_{\mathrm{eff} 2} \approx 0\right)$; as a result, the optical wave propagation in MSG-LD is like in BA LD - but the current flow (gain distribution) is modulated. The numerical results (Fig. 1) show that such design of BA is bound to increase the modal gain of the mode numbers 
close to the number of stripes. It is so because the optical field distribution in this mode is similar to the distribution of the gain. Additionally, the thermal lens effect, due to the self-heating of active regions, should help to achieve the generation of this high order mode, simultaneously preventing an optical coupling between neighbor stripes [9]. Active regions narrow enough should also prevent filamentation.

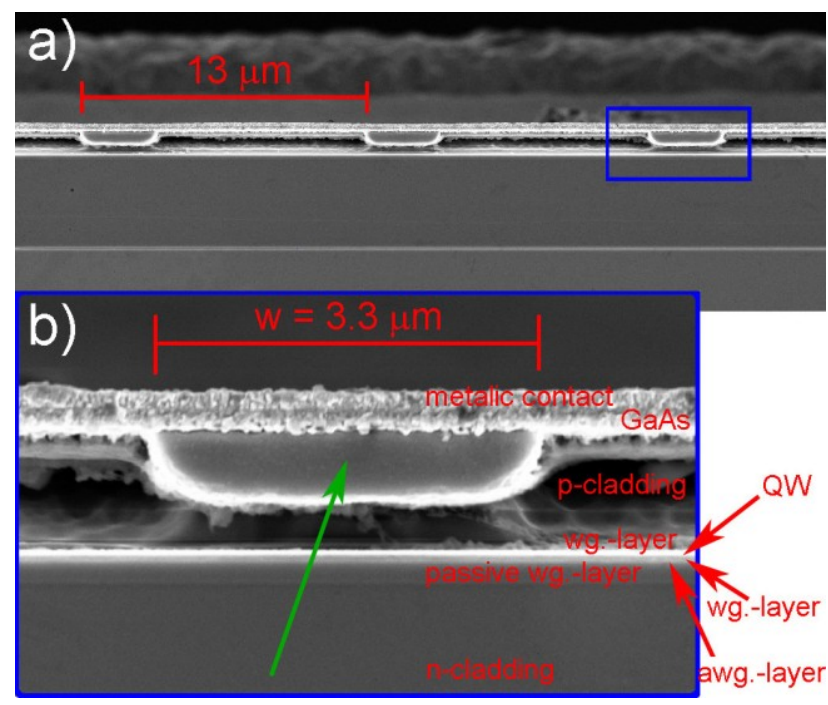

Fig. 2. The SEM microphotograph of a fragment of the MSG-LD structure (a) and zoom of the fragment marked by a blue rectangle (b). The green arrow shows the implanted region. The implantation depth is $\mathrm{h}=0.94 \mu \mathrm{m}$ and the waveguide layer distance from the surface is $1.2 \mu \mathrm{m}$. The thin layers: quantum well (QW), antiwaveguide layer (awg.layer) and $2^{\text {nd }}$ waveguide layer (wg.-layer) are marked by red arrows.

The asymmetric tensile-strained GaAsP/AlGaAs/GaAs heterostructure described previously [10] was used in this work. Such a heterostructure is designated for an 810-nm band and the asymmetric design allows obtaining a high catastrophic optical damage (COD) threshold.

The simplest way to achieve the designed gain distribution is the control of current flow distribution by the geometry of the p-contact. For this purpose, the $\mathrm{He}^{+}$ ion implantation through a previously evaporated p-contact was applied to define the current (gain) distribution. The used implantation mask consists of $\mathrm{N}=15$ narrow $(\mathrm{d}=10 \mu \mathrm{m})$ stripes which are placed in the center-to-center distance $\mathrm{S}=13 \mu \mathrm{m}$. The implantation energy and dose was $160 \mathrm{keV}$ and $5 \mathrm{e} 13 \mathrm{~cm}^{-2}$, respectively. Such conditions should ensure that the implant would not penetrate the waveguide layer. All the next technological processes are equal to these described previously [10].

The cavity length of the structures is equal to $\mathrm{L}=2 \mathrm{~mm}$. The number of stripes indicates that the $14^{\text {th }}$ order mode (the convention is that the fundamental mode is called $0^{\text {th }}$ order mode, the next mode is $1^{\text {st }}$ order mode etc.) should have the highest modal gain.

Figure 2a shows the SEM microphotograph of a fragment of the MSG-LD structure. At the zoomed part of this photograph (Fig. 2b), the implanted region marked by the green arrow and the layer sequence of the asymmetric heterostructure are shown. As it can be seen, the $\mathrm{He}^{+}$ions did not reach the waveguide (wg.-layer) and the widths of the implanted and unimplanted regions are obtained in good agreement with the mask.

The standard measurements of light power vs. current (L-C), near- and far-field characteristics in pulse conditions have been carried out. The threshold currents and the slope efficiencies of uncoated-mirrors MSG-LD are in the range of $\mathrm{I}_{\mathrm{th}}=1.14-1.2 \mathrm{~A}$ and $\eta=0.59-0.61 \mathrm{~W} / \mathrm{A}$, respectively. These results are slightly worse than the corresponding parameters of BA LD, but better than in the case of PLA. This suggests lower influence of losses introduced by $\mathrm{He}^{+}$implantation on the efficiency of MSG-LDs. The NF measurements were made in the standard experimental setup with a microscopic lens and a CCD camera. The FF characteristics were recorded in the experimental setup same as presented earlier [11].

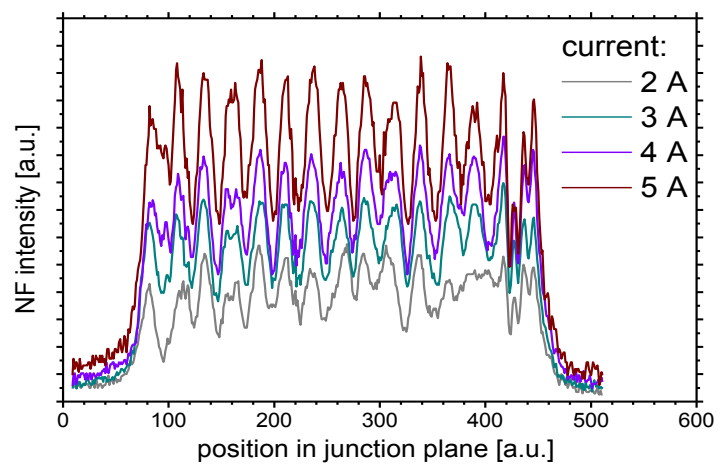

Fig. 3. Set of the NF distributions of the exemplary MSG-LD driven in a range of 2-5A. Plots show constant width and similar shape in the whole range of current drive. The number of maxima equal to the stripe number $(\mathrm{N}=15)$ suggests that the MSG structure maintained the $14^{\text {th }}$ order mode even at low currents.

Figure 3 shows the optical field distribution at the laser facet (NF) at different driving currents. The fifteen maxima typical of the $14^{\text {th }}$ order mode are visible even at low current $\mathrm{I}=2 \mathrm{~A}$. It a result of the $14^{\text {th }}$ order lateral mode lasing. It can be seen that the positions of maxima are the same at different currents, so good spatial stability is achieved. The constant width of an optical field and the number of maxima allow assuming that higher order modes (above $14^{\text {th }}$ ) are not maintained. Additionally, the more pronounced maxima at higher drive currents can be explained by the rising temperature of the active region. 


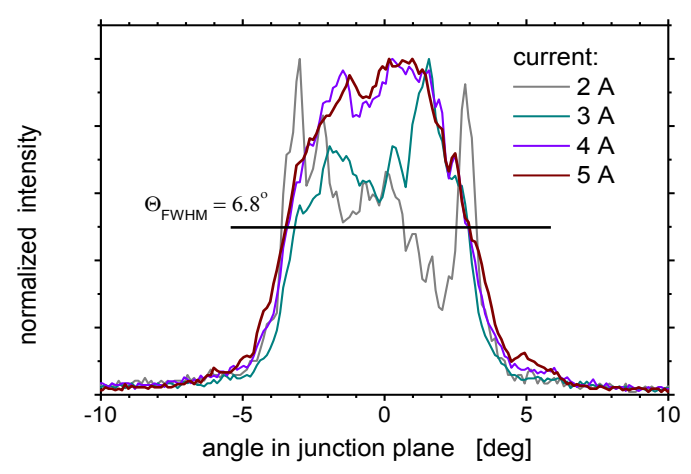

Fig. 4. The FF distribution of the MSG-LD (the same as shown in Fig. 3 ). The nearly constant FWHM and the disappearance of the supermodes at currents above $2 \mathrm{~A}$ are seen.

The optical field distribution in the far-field is seen in Fig. 4. At the low current $(\mathrm{I}=2 \mathrm{~A})$ there is quite a large difference in the intensity between the center and the sides of the distribution, which disappears at higher currents $(\mathrm{I}=4 \mathrm{~A}, 5 \mathrm{~A})$. It is connected partially with different threshold levels of the maintained modes (below $14^{\text {th }}$ ) and with the formation of supermodes like in PLA. Probably, the $\mathrm{He}^{+}$implantation leads also to form PLA with a weak coupling between the stripes. At currents above $\mathrm{I}=2 \mathrm{~A}$, thermal index guiding induced by self-heating causes an optical decoupling between the stripes and the whole structure starts to lase as BA LD. Lower-order modes fill the center of the beam distribution in the FF. The nearly constant width of this distribution confirms that the highest lasing mode in the MSG-LD is maintained at a low current. The full width at half maximum (FWHM) of the emitted beam is $\Theta_{\mathrm{FWHM}}=6.8^{\circ}$ and this value is almost constant over the whole measurements range. The $90 \%$ of the emitted optical power is contained within an angle of $\Theta_{\mathrm{P}=90 \%}=7.11^{\circ}$ at $\mathrm{I}=2 \mathrm{~A}$ and $\Theta_{\mathrm{P}=90 \%}=7.10^{\circ}$ at $\mathrm{I}=5 \mathrm{~A}$. This result has been compared with an analogous one for the standard BA LD (Fig. 5). Although the $\Theta_{\mathrm{P}=90 \%}$ of BA is smaller, the slow axis far-field blooming effect is stronger than in the case of MSG-LD. The differences between minimal and maximal widths are $\Delta=0.47^{\circ}$ and $0.9^{\circ}$ for MSG-LD and BA, respectively. It is expected that the beam with a constant divergence is easier for shaping than the narrower one but with a variable divergence. The narrowing of the beam in a range of $2-3 \mathrm{~A}$ is not clearly understood but it can be connected with vanishing of the supermodes.

In conclusion, the multi-stripe-gain laser diode (MSG-LD) structures have been presented. The experimental results show that the blooming effect is restricted. The current flow inducted by $\mathrm{He}^{+}$implantation leads to the $14^{\text {th }}$ order mode domination, which is in good agreement with numerical simulations.

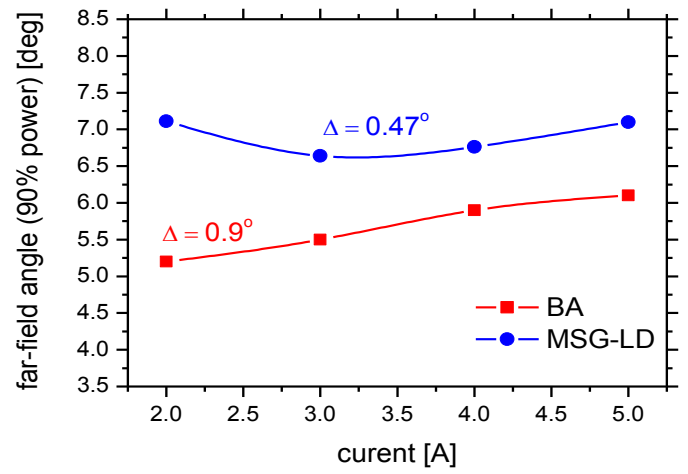

Fig. 5. The angle containing $90 \%$ of the power in an emitted beam at different currents for BA LD (red) and for MSG-LD (blue).

The MSG-LDs tend to operate with domination of the high order lateral mode also at the low drive current resulting in weaker variations of widths of the near- and far-field distributions compared to conventional broad area LDs. The maximal width of these distributions is reached slightly above the threshold current. It is expected that restricting the far-field blooming in the MSG-LDs will be better seen in $\mathrm{CW}$ operation, where the far-field blooming is mainly depending on thermal lens effect [12].

The work is supported by the research funds of the Polish National Center of Science under the grant number UMO2011/01/B/ST7/03333.

\section{References}

[1] J. Piprek, IEEE Photon. Technol. Lett. 25, 958 (2013).

[2] J. Piprek, Proc. SPIE 8619, 861910 (2013).

[3] P. Crump, S. Boeldicke, C.M. Schultz, H. Ekhteraei, H. Wenzel, G. Erbert, Semicond. Sci. Technol. 27, 045001 (2012).

[4] Y. Twu, K.L. Chen, A. Dienes, S. Wang, J.R. Whinnery, Electron. Lett. 21, 324 (1985).

[5] M.V. Maximov, Y.M. Shernyakov, I.I. Novikov, L.Ya. Karachinsky, N.Yu. Gordeev, U. Ben-Ami, D. Bortman-Abriv, A. Sharon, V.A. Shchukin, N.N. Ledentsov, T. Kettler, K. Posilovic, D. Bimberg, IEEE J. Sel. Topics Quantum Electron. 14, 1113 (2008).

[6] G. Sobczak, E. Dąbrowska, M. Teodorczyk, J. Kalbarczyk, A. Maląg, Proc. SPIE 8902, 890217 (2013).

[7] G. Sobczak, A. Maląg, Opto-Electr. Rev. 20, 134 (2012).

[8] G. Sobczak, E. Dąbrowska, M. Teodorczyk, J. Kalbarczyk, A. Maląg, Proc. SPIE 8702, 87020B (2013).

[9] M. Spreemann, B. Eppich, F. Schnieder, H. Wenzel, G. Erbert, IEEE J. Quantum Electron. 46, 1619 (2010).

[10] A. Maląg, E. Dąbrowska, M. Teodorczyk, G. Sobczak, A. Kozłowska, J. Kalbarczyk, IEEE J. Quantum Electron. 48, 465 (2012).

[11] A. Kozłowska, A. Maląg, Proc. SPIE 5120, 183 (2003).

[12] W. Sun, R. Pathak, G. Campbell, H. Eppich, J.H. Jacob, A. Chin and J. Fryer, Proc. SPIE 8605, 86050D-1 (2013). 